Supporting Information

\title{
Integration of Individual Nanoscale Structures into Devices using Dynamic Nanostenciling
}

\author{
Stefan Egger, Adelina Ilie, Shinichi Machida, Tomonobu Nakayama
}

\section{Experimental Methods}

The ultra-high vacuum (UHV) nanostencil instrument ${ }^{1,2}$ has inertial slider stages for coarse positioning in the $x-y$ plane and tilt alignment relative to the substrate, and a high resolution stage for in-plane movement (range: $100 \times 100 \mu \mathrm{m}^{2}$; capacitive sensor with $3 \mathrm{~nm}$ repeatability over full range). The sample is scanned relative to the tip holder. Materials are deposited using a multiple-cell electron beam evaporator.

The mask-cantilever is based on a commercially available AFM cantilever. Relatively large silicon cantilevers (SI-DF3, SII, Japan, dimensions: length $=460 \mu \mathrm{m}$; width $=$ $60 \mu \mathrm{m}$; thickness $=5 \mu \mathrm{m}$, nominal stiffness $1.9 \mathrm{~N} / \mathrm{m}$, resonance freq. $27 \mathrm{kHz}$, Rh coated) were chosen because they offer a sufficiently large area for masking and can be operated in non-contact AFM mode. The cantilevers were modified by FIB, as follows (see Figure 1B,C): with the highest ion beam current (etching rate for Si: $400 \mu \mathrm{m}^{3} / \mathrm{min}$ ) large apertures were cut, and two areas were thinned down to about $1 \mu \mathrm{m}$ thickness. Subsequently, a smaller beam intensity was chosen $(10-200 \mathrm{pA})$ for cutting small features in the thinned areas. For the cantilevers used in Figure 3, and Figure 5\&6 the tip was shortened to $2.5 \mu \mathrm{m}$ and $1.5 \mu \mathrm{m}$ in length, respectively. For the structures in Figure 4 an uncut, about $15 \mu \mathrm{m}$ long tip was used. The tip used for the electrical measurements from Figure $5 \& 6$ was rendered conductive by coating it in-situ with $\mathrm{Cr}$.

Imaging was implemented based on non-contact (frequency modulation detection) AFM, with optical detection. The sample is scanned relative to the tip holder. The cantilever oscillation amplitude was $30 \mathrm{~nm}$. Frequency set points between -1 and $-5 \mathrm{~Hz}$ and scanning speeds between 1 and $3 \mu \mathrm{m} / \mathrm{s}$ were used. The AFM images were processed by plane subtraction and flattened using the WSxM 2.1 freeware (www.nanotec.es).

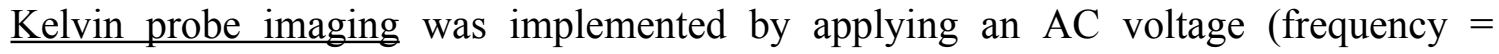
$200 \mathrm{~Hz}$, amplitude $=200 \mathrm{mV}$ ) on the cantilever and detecting the resulting amplitude in the frequency shift as the error signal. The frequency shift is measured with a bandwidth of $400 \mathrm{~Hz}$ using an EasyPLL plus detector from Nanosurf, Switzerland. A feedback loop added the appropriate DC voltage on the cantilever for minimizing the error signal (similar as in ref 3). This procedure is applied simultaneously (additional) to the normal nc-AFM technique, for which the dc-part of the frequency shift is used to follow the topography.

Potentiometry: A bias voltage of $100 \mathrm{mV}$ was applied on one device electrode, whereas the second electrode was grounded. The AFM tip was connected to a sourcemeter 
(Keithley 6430) to measure the potential along the device. Additionally, the contact resistance was checked at every point, by sourcing a small current ( $\pm 100 \mathrm{pA})$ while measuring the resulting voltage change. The contact resistance was found to be in the range of $0.5-20 \mathrm{M} \Omega$.

The SWCNTs were grown by the arc-discharge method, and then were dispersed using sonication in an aqueous surfactant (SDS) solution, followed by centrifugation for 10 hours at $15000 \mathrm{rpm}$. This lead to an aqueous solution enriched in individual SWCNTs. The nanotubes were dispersed on sapphire (0001) substrates, which were previously annealed in air at $1200{ }^{\circ} \mathrm{C}$ for $2 \mathrm{~h}$ to form atomically flat terraces ${ }^{4}$. To remove water from the surface the sample was subjected to annealing in UHV at $200{ }^{\circ} \mathrm{C}$ for one hour.

The porphyrin J-aggregates were formed in acidic aqueous solution ( $\mathrm{HCl}$ was added to reach $\mathrm{pH}$ 1) by self-assembly of porphyrin meso-tetrakis(4-sulfonatophenyl)porphine (TPPS4, concentration: $10^{-4} \mathrm{~mol} / \mathrm{l}$ ) (ref 5). A drop of the solution, containing the aggregates was dropped on the $\mathrm{SiO}_{2}(100 \mathrm{~nm}) / \mathrm{Si}$ substrate and, about $30 \mathrm{~s}$ later, spun at $5000 \mathrm{rpm}$.

The photoconductivity measurements were performed ex-situ with the sample mounted in Ar atmosphere and using an optical microscope equipped with a laser at $\lambda=488 \mathrm{~nm}$. To facilitate homogenous illumination of the device the laser was defocused on the substrate to a spot size of about $50 \mu \mathrm{m}$ in diameter. A bias of $1 \mathrm{~V}$ was applied to the device in dual terminal configuration. The illumination was switched on while slowly increasing the light intensity from 0.5 to $20 \mathrm{~kW} / \mathrm{m}^{2}$ using a set of neutral density filters.

Nano-gap series: The nano-gaps were formed between Ti and Pd electrodes, which were fabricated using the same cantilever/mask, but with two different apertures cut in this mask: The first aperture defined the Ti electrode; the second aperture defined the $\mathrm{Pd}$ electrode. The nano-gaps were fabricated in the order: Ti electrode of gap structure no.1, $\mathrm{Pd}$ electrode of gap structure no.1, Ti electrode of gap structure no.2, etc. The apertures had a rectangular end, $290 \mathrm{~nm}$ wide and of $25 \mathrm{~nm}$ corner radius.

The width of the wedge of the evaporated structures that occurs due to the shadow produced by the cantilever-mask can be approximated by $D \cdot(a / b) \approx 44 \mathrm{~nm}$, where $D \approx$ $3.5 \mathrm{~mm}$ is the diameter of the evaporator's crucible, $b \approx 20 \mathrm{~cm}$ is the crucible-tocantilever distance, while $a \approx 2.5 \mu \mathrm{m}$ is the mask-to-substrate distance. $a$ is given by the length of the cantilever's tip, and as discussed in the text, shorter tips produce better edge definition. The precise profile of the edge can be calculated as the convolution of the evaporator's beam profile and the shape of the mask's aperture. In our case, as the nano-gaps are defined using the corners of the apertures, it is the corner radius that affects the edge sharpness. The profiles from Figure 3E (blue, green lines) were calculated using the dimensions given above. The comparison of the various nano-gaps in the series could be affected by an additional, cumulative effect: the aperture edges could progressively change during the fabrication sequence due to material adsorbed on the cantilever/mask (clogging). This could lead to a progressive loss of definition of the resulting electrodes. The shape change due to clogging is estimated to be between 0.1 and 0.3 times the thickness of the evaporated layers ${ }^{6}$. As our electrodes are only about $3 \mathrm{~nm}$ thick this effect is negligible for the nano-gap series discussed here. Indeed, 
experimentally we found that the $\mathrm{Pd}$ (Ti) electrode of the gap structure no.3, i.e. the last one to be fabricated in the series, is at most $3 \mathrm{~nm}$ smaller (as given by the limited accuracy of AFM measurements) than the Pd (Ti) electrode of gap structure no.1, i.e. the first one to be fabricated in the series. Therefore, we attribute the differences between the experimental and calculated edge profiles mainly to the effect of surface diffusion of the evaporated materials.

\section{Estimation of gap sizes}

The estimation of the gap size from Figure 3 from AFM images is affected by the convolution with the tip shape and the specifics of the forces involved during tapping mode, usually leading to a smaller gap than the real value. An upper limit for the gap $\mathrm{w}_{1}$ can be obtained based on the comparison between the AFM measured sizes of the gaps in the series from Figure 3 and the corresponding inter-gap resistances. Based on the respective AFM images (see Figure S1) we found: $w_{3}=w_{1}-20 \mathrm{~nm}$, and $w_{2}=w_{3}-$ $17 \mathrm{~nm}$. The in-situ gap resistances $R_{1}=8 \mathrm{~T} \Omega ; R_{2}<1 \mathrm{M} \Omega ; R_{3}=0.5 \mathrm{G} \Omega$ were measured with a $\mathrm{Si}$ tip with a contact resistance on a Pd surface of about $1 \mathrm{M} \Omega$. These values allowed us to estimate that the overlapping structure no.2 is, as expected, fully conductive, while structure no. 3 has a gap $w_{3} \approx 0 \mathrm{~nm}$, leading to $w_{1} \approx 20 \mathrm{~nm}$.

\section{Surface diffusion during material evaporation}

Surface diffusion is the ultimate factor limiting structure resolution (sharpness) in nano-stencilling ${ }^{2}$. The importance of diffusion processes at ambient temperature is strongly material dependent and varies over many orders of magnitude 7 . Fig. 3E shows that the profile of the Pd electrode is significantly widened, by about $40 \mathrm{~nm}$. Such a widening requires diffusion of $\mathrm{Pd}$ atoms on top of Pd (self-diffusion). STM studies showed that single Pd atoms can diffuse on a Pd(111) surface, whereas clusters with two or more atoms are stable at room temperature ${ }^{8}$ and serve as nuclei for the growth of larger islands. The resulting island density was given as $1.4 \times 10^{-5}$ islands per adsorption site $^{8}$, which corresponds to about one island per $70 \times 70 \mathrm{~nm}^{2}$. From this we can estimate the typical length scale for the relocation of freshly adsorbed atoms to be of the order of several tens of $\mathrm{nm}$. The defect free $\operatorname{Pd}(111)$ surface has the highest diffusivity ${ }^{9}$ and is therefore the worst case scenario leading to loss of structure resolution. In our case, it is realistic to assume a rather disordered film structure, where the mean free path of the diffusing atoms is expected to be reduced by defects, leading to a smaller average relocation.

For the Ti electrodes diffusion is significantly smaller. We found this systematically, in all our experiments and with different types of substrates; this appears to also be in agreement with the higher melting temperature and higher cohesion energy of $\mathrm{Ti}$ compared to $\mathrm{Pd}$ (ref 10). In addition to self diffusion, diffusion of metal atoms or clusters on the bare $\mathrm{SiO}_{2}$ substrate could also lead to undesired effects, such as conductive paths. Nevertheless we did not find evidence for diffusion beyond the mask aperture area, taking into account the AFM images (Figure 3) and the high resistance of the $20 \mathrm{~nm}$ gap. 
Note that in the considerations above we focused entirely on the diffusion of the freshly arriving mobile atoms, whereas diffusion which requires the creation of mobile defects ("mesoscale surface diffusion" or "mass transfer surface diffusion") was neglected. This assumption seems to be appropriate because (i) the diffusion constants for mesoscale surface diffusion are expected to be very small $\left(\sim 10^{-19} \mathrm{~cm}^{2} / \mathrm{s}\right.$ for $\mathrm{Pd}$ at $298 \mathrm{~K}$, see ref 10,11) and (ii) we have never observed changes in the shapes of the Pd or Ti structures after material evaporation.

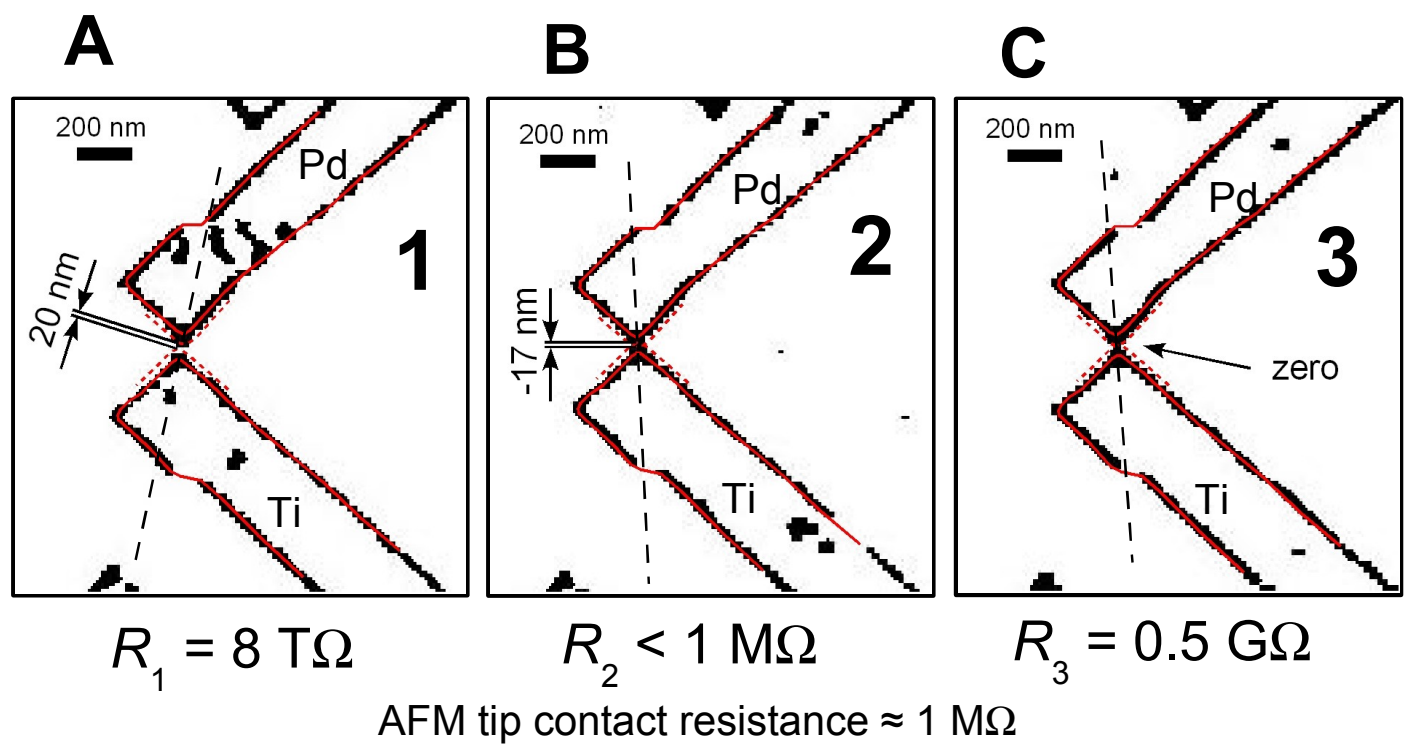

Figure S1. Calculation of relative electrode positions for the nanogap series. $(\mathrm{A}, \mathrm{B}, \mathrm{C})$ are images of the three gap structures $(1,2,3)$ from Figure $3 \mathrm{~A}$. These images were processed by calculating the second derivative in the fast scanning direction (horizontal in this figure), and adjusting the grey levels for high contrast. The image processing was done on the overall image, before marking the three gap regions. The red contour line was drawn manually along the electrodes in (A), copied into (B) and (C), and moved in order to match the position of the electrodes. These contour lines do not show the true electrode edges (for comparison, see the direct topography images shown in Figure 3), but differences between these contours in the three images above are expected to be the same as the relative differences between the true nanogaps in the series from Figure 3. Based on the conductivity measurements, we assume that for device no.3 the electrodes are approximately touching (gap size $=0$ ) ( see also main text). Consequently, gap no.1 was calculated to be $20 \mathrm{~nm}$, while the overlap of the structure no.2 to be $17 \mathrm{~nm}$. The three gap structures were fabricated in the order shown by the numbers. In-situ imaging after the fabrication of each gap structure allowed us to correct position errors, to make structures no. 2 and 3 better horizontally aligned than no.1 (the dashed line is more vertical). 


\section{Estimation of temperature change caused by evaporator}

Thermal evaporation requires heating of the source material to temperatures of the order of $1000{ }^{\circ} \mathrm{C}$ (for example, $T_{\text {evap,Ti }} \approx 1500{ }^{\circ} \mathrm{C}, T_{\text {evap,Pd }} \approx 1200{ }^{\circ} \mathrm{C}$ ). The glowing material produces a considerable amount of thermal radiation, while the substrate is positioned relatively close, at about $20 \mathrm{~cm}$ distance. How significant is the resulting temperature increase of mask and substrate?

The total heating power $P$ of the evaporator during structure fabrication is typically in the range 30 to $150 \mathrm{~W}$, depending on the material (for example, $P_{\mathrm{Ti}} \approx 100 \mathrm{~W}, P_{\mathrm{Pd}} \approx$ $60 \mathrm{~W}$ ). The power density of the thermal radiation reaching the mask/substrate can be roughly estimated as $p \approx P /\left(4 \pi b^{2}\right) \approx 20 \mathrm{~mW} / \mathrm{cm}^{2}$ (with $P=100 \mathrm{~W}$, evaporator-to-mask distance $b=20 \mathrm{~cm}$, and assuming an isotropic distribution of the radiation) $)^{\dagger}$.

The energy absorbed by the cantilever

$P_{\text {absorbed }}=p(1-\rho) A_{\text {exposed }}$

where $\rho \approx 0.5$ is the reflectivity, and $A_{\text {exposed }}=L_{1} \times w=120 \times 60 \mu \mathrm{m}^{2}$ is the area of the cantilever exposed to radiation, dissipates along the cantilever to a large reservoir. The expected temperature increase is

$\Delta T=P_{\text {absorbed }} L /\left(h w k_{\mathrm{Si}}\right)$

where $L \approx 400 \mu \mathrm{m}, h=5 \mu \mathrm{m}$, and $w=60 \mu \mathrm{m}$ are the cantilever's dimensions, while $k_{\mathrm{Si}}$ $\approx 150 \mathrm{Wm}^{-1} \mathrm{~K}^{-1}$ is the thermal conductivity. The above values give a temperature increase of $\Delta T \approx 0.006 \mathrm{~K}$.

Condensation of 'hot' metal atoms landing on a surface can also contribute to the energy absorbed by the cantilever. The total enthalpy change from hot vapor to room temperature solid is about $5 \mathrm{eV} /$ atom (cohesion energy of Ti is $4.85 \mathrm{eV}$, see ref 12, while the kinetic energy of the impinging atoms is less than $1 \mathrm{eV}$ ). An evaporation rate of $1 \mathrm{~nm} / \mathrm{min}\left(\sim 6 \times 10^{15}\right.$ atoms $\left.\mathrm{cm}^{-2} \mathrm{~min}^{-1}\right)$ gives a power density of about $0.1 \mathrm{~mW} / \mathrm{cm}^{2}$. This is far less than the power adsorbed by the cantilever directly from thermal radiation, which therefore appears to be the main factor that could lead to a temperature increase of the cantilever.

We evaluated the cantilever's temperature increase experimentally by monitoring the change of the cantilever resonance frequency. Using $\Delta f=-5.8 \times 10^{-5} \times f_{0} \times \Delta T$ from ref 13 and $f_{0}=27 \mathrm{kHz}$, the resonance frequency of our cantilever, a temperature increase of $1 \mathrm{~K}$ would decrease the resonance frequency by about $1.6 \mathrm{~Hz}$. Similarly, from the frequency variations recorded in Figure 2 after closing the evaporator's valve, we estimate $\Delta T<0.05 \mathrm{~K}$.

The temperature increase of the substrate is expected to be smaller than that of the cantilever because the exposed area is only about ten times bigger (circular area, see Figure 1D) while the thermal conductance is much higher.

In summary, the temperature increase of the cantilever and substrate during evaporation is negligible. 


\section{References}

(1) Egger, S.; Ilie, A.; Fu, Y. T.; Chongsathien, J.; Kang, D. J.; Welland, M. E. Nano Lett. 2005, 5, 15-20.

(2) Egger, S.; Higuchi, S.; Nakayama, T. J. Comb. Chem. 2006, 8, 275-279.

(3) Loppacher, C.; Zerweck, U.; Teich, S.; Beyreuther, E.; Otto, T.; Grafstrom, S.; Eng, L. M. Nanotechnology 2005, 16, S1-S6.

(4) Yoshimoto, M.; Maeda, T.; Ohnishi, T.; Koinuma, H.; Ishiyama, O.; Shinohara, M.; Kubo, M.; Miura, R.; Miyamoto, A. Appl. Phys. Lett. 1995, 67, 2615-2617.

(5) Rotomskis, R.; Augulis, R.; Snitka, V.; Valiokas, R.; Liedberg, B. J. Phys. Chem. B 2004, 108, 2833-2838.

(6) Lishchynska, M.; Bourenkov, V.; van den Boogaart, M. A. F.; Doeswijk, L.; Brugger, J.; Greer, J. C. Microelectron. Eng. 2007, 84, 42-53.

(7) Brune, H. Surf. Sci. Rep. 1998, 31, 121-229.

(8) Steltenpohl, A.; Memmel, N. Surf. Sci. 2000, 454, 558-561.

(9) Agrawal, P. M.; Rice, B. M.; Thompson, D. L. Surf. Sci. 2002, 515, 21-35.

(10) Gjostein, N. A., Short Circuit Diffusion. In Diffusion, Aaronson, H. I., Ed. American Society for Metals: Metals Park, Ohio, 1973; p 241-274.

(11) Beszeda, I.; Gontier-Moya, E. G.; Beke, D. L. Surf. Sci. 2003, 547, 229-238.

(12) Kittel, C., Introduction to Solid State Physics. $8^{\text {th }}$ ed.; Wiley: New York, 2005.

(13) Giessibl, F. J. Rev. Mod. Phys. 2003, 75, 949-983. 